\title{
Deficits in analogical reasoning in adolescents with traumatic brain injury
}

\section{Daniel C. Krawczyk ${ }^{1,2 *}$, Gerri Hanten ${ }^{3}$, Elisabeth A. Wilde ${ }^{3,4,5}$, Xiaoqi Li ${ }^{3}$, Kathleen P. Schnelle ${ }^{3}$, Tricia L. Merkley ${ }^{5}$, Ana C. Vasquez ${ }^{3}$, Lori G. Cook', Michelle McClelland' ', Sandra B. Chapman' and Harvey S. Levin ${ }^{3,6}$}

\author{
' Center for Brain Health, School of Behavioral and Brain Sciences, The University of Texas at Dallas, TX, USA \\ 2 Department of Psychiatry, University of Texas Southwestern Medical Center at Dallas, TX, USA \\ ${ }_{3}$ Physical Medicine and Rehabilitation Alliance of Baylor College of Medicine and the University of Texas-Houston Medical School, Houston, TX, USA \\ ${ }^{4}$ Departments of Radiology and Neurology, Baylor College of Medicine, Houston, TX, USA \\ ${ }^{5}$ Department of Psychology, Brigham Young University, Provo, UT, USA \\ ${ }^{6}$ Department of Neurosurgery, Baylor College of Medicine, Houston, TX, USA
}

\section{Edited by:}

Silvia A. Bunge, University of California Berkeley, USA

\section{Reviewed by:}

Lindsey Richland, University of

California Irvine, USA

Miriam Beauchamp, Université de

Montréal, Canada

${ }^{*}$ Correspondence:

Daniel C. Krawczyk, Center for Brain

Health, The University of Texas at

Dallas, 2200 Mockingbird Lane, Dallas,

TX 75235, USA

e-mail:daniel.krawczyk@utdallas.edu
Individuals with traumatic brain injury (TBI) exhibit deficits in executive control, which may impact their reasoning abilities. Analogical reasoning requires working memory and inhibitory abilities. In this study, we tested adolescents with moderate to severe TBI and typically developing (TD) controls on a set of picture analogy problems. Three factors were varied: complexity (number of relations in the problems), distraction (distractor item present or absent), and animacy (living or non-living items in the problems). We found that TD adolescents performed significantly better overall than TBI adolescents. There was also an age effect present in theTBI group where older participants performed better than younger ones. This age effect was not observed in the TD group. Performance was affected by complexity and distraction. Further, TBI participants exhibited lower performance with distractors present thanTD participants. The reasoning deficits exhibited by the TBI participants were correlated with measures of executive function that required working memory updating, attention, and attentional screening. Using MRI-derived measures of cortical thickness, correlations were carried out between task accuracy and cortical thickness. The TD adolescents showed negative correlations between thickness and task accuracy in frontal and temporal regions consistent with cortical maturation in these regions. This study demonstrates that adolescent TBI results in impairments in analogical reasoning ability. Further, TBI youth have difficulty effectively screening out distraction, which may lead to failures in comprehension of the relations among items in visual scenes. Lastly, TBI youth fail to show robust cortical-behavior correlations as observed in TD individuals.

Keywords: analogy, reasoning, traumatic brain injury, gray matter, adolescent, distraction

\section{INTRODUCTION}

Traumatic brain injury (TBI) stands as one of the chief health problems facing today's youth. It is the most frequent cause of death and disability among children in the United States (Langlois et al. $2003,2005)$. There are a variety of cognitive deficits that frequently result from TBI including both executive functioning deficits (Levin et al., 1994; Dennis et al., 2001; Ewing-Cobbs et al., 2004) and social deficits (Taylor et al., 2002; Yeates et al., 2007; Hanten et al., 2008). Specific cognitive functions necessary for human reasoning include processing abstract information and screening out distraction. The current study investigates the effect of TBI upon the reasoning ability of adolescents.

Reasoning has been assessed across development and findings have revealed that reasoning abilities mature in parallel with broader increases in executive function abilities. Critical to reasoning capacity, a relational shift has been described in which individuals move from reasoning about surface features and perceptual similarity toward reasoning about relations among elements (Brown, 1989; Gentner and Rattermann, 1991; Goswami and Brown 1989; Kotovsky and Gentner, 1996). This shift occurs when sufficient relational knowledge has been acquired, which is frequently observed in childhood, but may occur at any point developmentally when the appropriate knowledge has been obtained. This shift in focus allows individuals to consider abstract relations among items when solving problems. This capacity appears to be unique to humans (Penn et al., 2008) and is believed to underlie the extended reasoning abilities that humans have over other species (Halford et al., 1994; Holyoak and Thagard, 1996). Relational reasoning abilities have been studied using visual-spatial matrix problems such as those found in the Raven's progressive matrices (Raven, 1938). These capacities have been shown to follow a developmental time-course such that individuals typically reach higher levels of competence by adolescence (Sternberg and Rifkin, 1979; Crone et al., 2009).

\section{REASONING BY ANALOGY}

Solving analogies has been considered to be a paramount accomplishment in relational reasoning (Gentner, 1983; Hummel and Holyoak, 2003).Analogy performance requires the ability to understand relations among two or more elements in a source analog 
and to map that relation onto other relations present in a target analog. These capacities develop across childhood (Goswami and Brown, 1989; Gentner and Rattermann, 1991; Goswami et al., 1998; Rattermann and Gentner, 1998; Goswami, 2001) as the ability to understand similarity improves (Goswami and Brown, 1989; Goswami et al., 1998). Another key factor relevant to analogy performance is working memory (WM) function with increases in WM capacity leading to concurrent expansion of relational reasoning abilities (Waltz et al., 2000; Morrison et al., 2004). Achieving successful solutions to analogy problems places high demand upon WM and may also invoke inhibitory control processes under conditions when perceptual or feature similarities between individual objects exist (Gentner and Toupin, 1986). Such conditions have been demonstrated to increase processing time and error rates in analogy problems (Morrison et al., 2004; Viskontas et al., 2004).

A clear form of analogy problems in which relational and perceptual feature similarity compete is in picture analogies. Markman and Gentner (1993) tested participants on a set of scene analogy problems that included relational matches as well as perceptual distractors. Solutions to such problems were demonstrated to be dependent upon executive WM in a dual-task study (Waltz et al., 2000). A more extensive set of picture analogy problems was recently developed by Richland et al. (2006). These investigators varied relational complexity of the analogies finding that problems involving two-relations were more difficult than those that involved only one relation. Additionally, the presence of perceptual distractors was varied such that problems with distractors were solved at lower levels than those without distractors. Developmentally, children ranging in age from 3- to 7-years old were not as competent as 13- to 14-year-old adolescents. Thus, analogical reasoning abilities increase with age and depend upon WM abilities that enable relational processing and inhibitory control over competing perceptual alternative matches.

Theoretical models of analogical reasoning have implicated executive control functions as being central to the analogical mapping process. Learning and inference with schemas and analogies (LISA) is a model that has emphasized that the limits of working memory also limit the ability to map elements of analogous situations together (Hummel and Holyoak, 2003). Further, Hummel and Holyoak (1997) demonstrated that developmental limits on analogy can be simulated by reducing the working memory capacity of that model. Broader models of cognition, such as ACT-R have further linked analogical reasoning to working memory processes through emphasizing active memory retrieval (Salvucci and Anderson, 2001). These theoretical models provide motivation to test the degree to which executive functions are related to analogical reasoning in the current investigation.

Networks involving the prefrontal cortex (PFC), the temporal cortex, and the parietal lobes may be particularly relevant to analogical reasoning based on prior findings in populations with frontotemporal lobar degeneration (Morrison et al., 2004; Krawczyk et al., 2008). The results of TBI working memory studies suggest that older children and early adolescents may have deficits in n-back task performance (Levin et al., 2002, 2004) and that WM performance is related to injury severity and time post-injury with children sustaining mild to moderate TBI showing little change over time, whereas children with severe TBI perform worse overall, and show a decline in performance with time. Evidence from neuroimaging suggests that adolescents with moderate to severe TBI show over-activation of PFC and parietal regions related to maintenance of information in WM relative to typically developing (TD) youth, and that this may represent an inefficient over-recruitment of cortical activity in compensation for the brain injury (Newsome et al., 2008).

Inhibitory control deficits in working memory are likely to be particularly problematic for the reasoning abilities of individuals with TBI, as inhibitory deficits have been strongly implicated in failures of reasoning in adults (Viskontas et al., 2004; Richland et al., 2006). Dennis et al. (2001) demonstrated that children with TBI had difficulties screening out irrelevant digit information in a vigilance task. Other studies have reported inhibitory control deficits in TBI individuals in go-no-go tasks (Konrad et al., 2000a,b), stop-signal tasks (Schachar et al., 2004), and shifting tasks (Levin et al., 1993).

\section{EXECUTIVE FUNCTION IN TRAUMATIC BRAIN INJURY}

Adolescents with TBI frequently have disruptions of the frontal networks associated with executive functions relevant to reasoning (Levin and Hanten, 2005). This leads to deficits in working memory and inhibitory control. Such deficits may also lead to disabilities with abstract reasoning. MRI studies of children with TBI (Levin et al., 1997; Wilde et al., 2005) have supported this link revealing both focal lesions and diffuse injury, particularly to the prefrontal, frontal, and temporal regions relevant to cognitive control. Prefrontal regions are vulnerable due to their proximity to the sphenoidal ridges and bony protrusions on the base of the skull and gray matter in these regions has been found to be reduced even in the absence of focal lesions (Berryhill et al., 1995). MRI studies conducted several months to years post-injury have shown that loss of white matter can be extensive (Wilde et al., 2005; Ghosh et al., 2009; Bigler et al., 2010). Diffuse cortical thinning has been reported in children with moderate to severe TBI as compared to typically developing children (Merkley et al., 2008) or children with orthopedic injury (McCauley et al., 2010). Thus both gray and white matter disruptions likely contribute to the working memory deficits observed in TBI adolescents. While there is little current literature on relational reasoning after TBI, the presence of executive function deficits in TBI predict that they may be impaired on analogical reasoning. TBI has been demonstrated to decrease performance on the Raven's progressive matrices task (Hiscock et al., 2002), which is a relational reasoning task closely linked to analogical reasoning performance (Morsanyi and Holyoak, 2010; Geake and Hansen, 2010). Given the declines after TBI in both basic executive functioning (Levin and Hanten, 2005; Muscara et al., 2008) and in Raven's matrices (Hiscock et al., 2002), it is predicted that adolescents with TBI will have decreases in analogical reasoning performance. Additionally, the evidence of inhibitory control declines after TBI support the prediction that distractor items are likely to decrease performance on picture analogies (Leblanc et al., 2006; O’Keeffe et al., 2007).

We compared adolescents with TBI and control participants who were TD youth on analogical reasoning and executive function tasks. Participants completed a battery of scene analogies based on a set previously developed by Richland et al. (2006). We manipulated the number of relations relevant to solving the analogies (onerelation versus two-relation). We predicted that both groups would 
show differences in relation numbers, but that TBI participants will show greater deficits in response to the greater relational demand, as these participants are likely to have both WM maintenance and executive control deficits (Levin et al., 2002, 2004). We also tested for the effect of distractors upon reasoning performance in TBI participants. We predicted that TBI participants would exhibit deficits in analogical reasoning due to their tendency to have reduced cortical thickness related to executive functioning (Levin et al., 2008). This prediction follows from prior studies indicating that executive control deficits lead to failures of reasoning in young children (Richland et al., 2006) and older adults with dementia (Morrison et al., 2004; Krawczyk et al., 2008). Lastly, we modified the problem set to compare living and non-living analogy problems. We included an exploratory social component in the current study by varying the type of problems so that some analogies focus on relations among living elements, emphasizing intentions, and cooperation, while others focus on relational correspondences among inanimate objects, emphasizing spatial relationships. This was included to test whether TBI adolescents would show selective deficits in reasoning about relationships among living items based on prior evidence of their deficits in perceiving social situations (Janusz et al., 2002; Yeates et al., 2002; Hanten et al., 2008).

We administered two executive function tasks in order to assess the degree to which WM updating and inhibitory control abilities interact with reasoning performance. These tasks assess the ability to update WM and to allocate attention strategically toward high priority information while screening out lower priority information. These skills were predicted to be important for analogical reasoning, as prior evidence suggests that executive WM dual tasks disrupt the ability to produce solve picture analogies (Waltz et al., 2000). Similar deficits have been observed in picture analogy performance under high anxiety conditions that would likely reduce WM resources (Tohill and Holyoak, 2000). We predicted that executive control abilities within WM would be predictive of performance on the analogical reasoning task and may underlie the performance deficits predicted to be present in TBI adolescents particularly when problem complexity is higher and distraction must be screened out.

We also collected MRI data from the participants in order to assess cortical thickness related to the analogy task. While there is little literature describing the relationship between morphometry data and analogical reasoning, prior studies of analogy using functional MRI have demonstrated activation of frontal, parietal, and temporal cortex, often with a left hemisphere asymmetry (Wharton et al., 2000; Bunge et al., 2005, 2009; Luo et al., 2003; Green et al., 2010). Several studies have indicated that the frontal pole is particularly important for relational responding (Smith et al., 2007; Christoff et al., 2009; Wendelken et al., 2008). Studies of relational reasoning in patients with frontotemporal lobar degeneration have also demonstrated the necessity of intact PFC for matrix reasoning, transitive reasoning (Waltz et al., 2000), verbal analogy, and visual analogy performance (Morrison et al., 2004; Krawczyk et al., 2008). Notably, individuals with PFC damage caused by dementia demonstrated additional impairments when distraction needed to be screened out in these prior studies. Furthermore, temporal cortex was implicated as relevant in these studies based on its central role in semantic memory (Morrison et al., 2004; Krawczyk et al., 2008; Morrison and Soohyun, 2008). We predicted that healthy control participants would show relationships between cortical thickness and analogy performance in the temporal cortex and regions in the lateral PFC including the left frontal pole.

\section{MATERIALS AND METHODS PARTICIPANTS}

Twelve youth who sustained a moderate-severe TBI (MSTBI) at least 1 year prior were recruited from a cohort of children with TBI, specifically from the Baylor College of Medicine and University of Texas at Dallas sites ( 7 males and 5 females; age at test: $M=16.51$ years, $\mathrm{SD}=2.14$, range 12.79-19.12; Glasgow coma scale (GCS) score: $M=7.33, \mathrm{SD}=3.89$, range 3-15; post-injury interval: $M=2.65$ years, $\mathrm{SD}=0.76$, range $0.94-3.62$; parental education: $M=12.83$ years, $\mathrm{SD}=3.32$, range $6-16$; ethnicity: 7 Caucasian, 1 African-American, 4 Hispanic). Severe TBI involved a non-penetrating TBI arising from closed head trauma (i.e., acceleration, deceleration, or impact by a blunt object) with lowest postresuscitation GCS score of 3-8 indicative of coma that is not due to child abuse and is not complicated by hypoxia (PO2 $<96 \mathrm{mmHg}$ or systolic blood pressure $\geq 2$ SDs below mean for age) for $30 \mathrm{~min}$ or longer after resuscitation. In cases of delayed neurologic deterioration, the worst GCS score was be used. Following convention (Teasdale and Jennett, 1974), the best level of response recorded for each component of the GCS at a specific time was used to obtain the sum GCS score. Injury variables, including in-hospital CT scan and pupillary response were recorded (Saatman et al., 2008). Other inclusion criteria were age 12-18 years, right handedness (Oldfield, 1971) to mitigate heterogeneity in brain asymmetries, cognitive ability to assent or consent (depending on age). The classification of moderate TBI differed from severe TBI by a lowest post-resuscitation GCS score of 9-12, indicating impaired consciousness but not coma (Teasdale and Jennett, 1974) or a GCS score of 13-15 with an acute brain lesion on the CT scan within $24 \mathrm{~h}$ after injury. Patients with brain lesions despite mild impairment of consciousness were included because they frequently exhibit cognitive deficit at 12 months post-injury (Levin et al., 2008). Other injury variables were recorded as described for severe TBI. Table 1 shows lesion location, GCS, and type of accident for TBI participants, 10 of whom had clear lesions. Eleven TD children were recruited

\section{Table 1 | Injury characteristics of TBI Participants who had evidence of} focal lesions.

\begin{tabular}{lrll}
\hline Subject & GCS & Mechanism & Lesion location(s) \\
\hline 1 & 10 & Auto & Occipital, parietal, temporal \\
2 & 7 & Auto & Occipital, temporal, corpus callosum \\
3 & 3 & Auto & No lesion \\
4 & 9 & Fall & Frontal \\
5 & 3 & Fall & Frontal, temporal, basal ganglia \\
6 & 15 & Fall & Frontal, temporal \\
7 & 7 & Fall & Frontal, parietal, temporal \\
8 & 8 & Auto & Unclear \\
9 & 3 & Auto & Frontal, occipital, corpus callosum \\
10 & 3 & Fall & Frontal, parietal, temporal \\
11 & 12 & Fall & Frontal, parietal, temporal \\
12 & 8 & Auto & Temporal
\end{tabular}


from our cohort of healthy subjects in the local community (6 males and 5 females; age: $M=16.37$ years, $\mathrm{SD}=1.89$, range 13.94-19.24; parental education: $M=15$ years, $\mathrm{SD}=2.37$, range 11-19; ethnicity: 6 Caucasian, 1 African-American, 4 Hispanic). There were no significant group differences in gender, Fisher's exact test $p=1$; age, $t(21)=0.17, p=0.8702$; parental education, $t(21)=-1.87, p=0.0888$, or ethnicity, Fisher's exact test, $p=1$. Informed consent was obtained prior to the experiment for all participants. Experiments were approved by the Institutional Review Boards at Baylor College of Medicine and The University of Texas at Dallas.

\section{PICTURE ANALOGY EXPERIMENT}

Materials

The set of analogies was developed from prior scene analogy experiments (Richland et al., 2006). The analogies consisted of two pictures of scenes oriented vertically (refer to Figure 1). Each scene consisted of five items that were objects, people, or animals. Two or three of these items were involved in a relationship, such as chasing, pulling, etc. One item in the source scene (top picture) had a black arrow pointing to it. This item was to be matched to a similar item in the target scene (bottom picture) in order to complete an analogy between the top and bottom scenes. The prior task (Richland

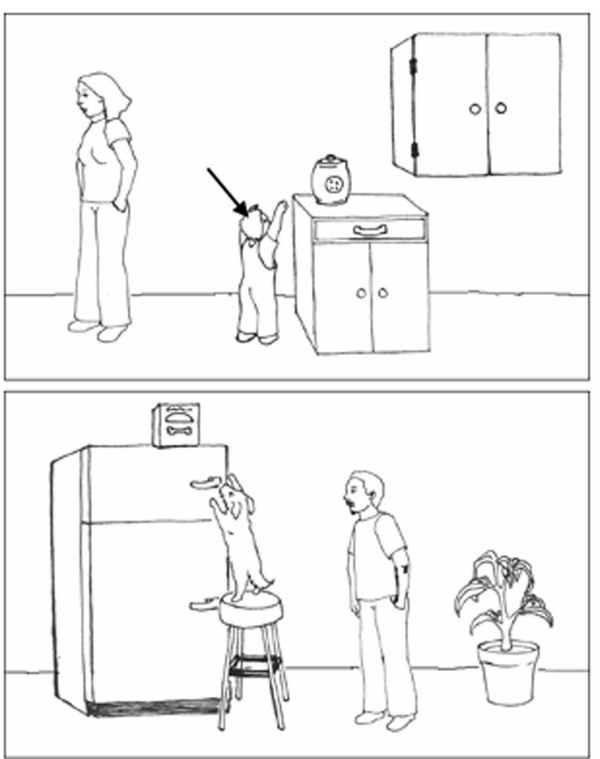

A. Living, one-relation, no distractor

C. Non-living, one-relation, distractor
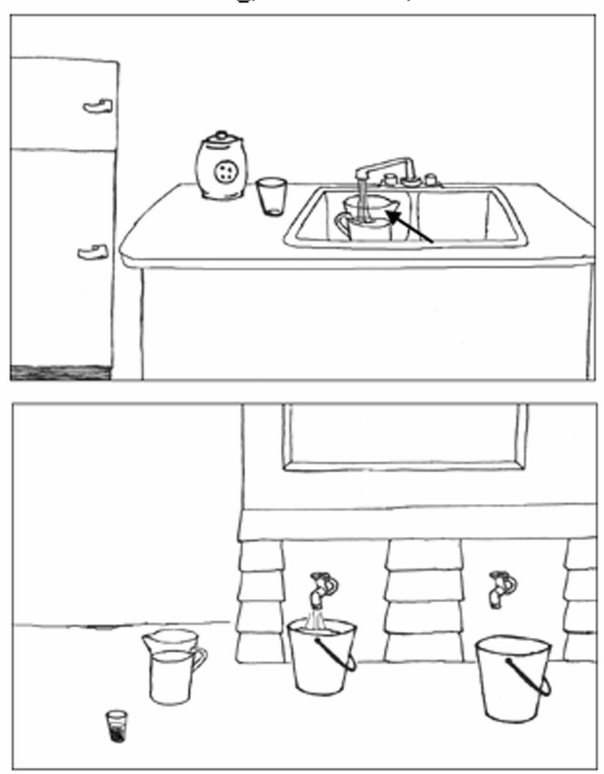
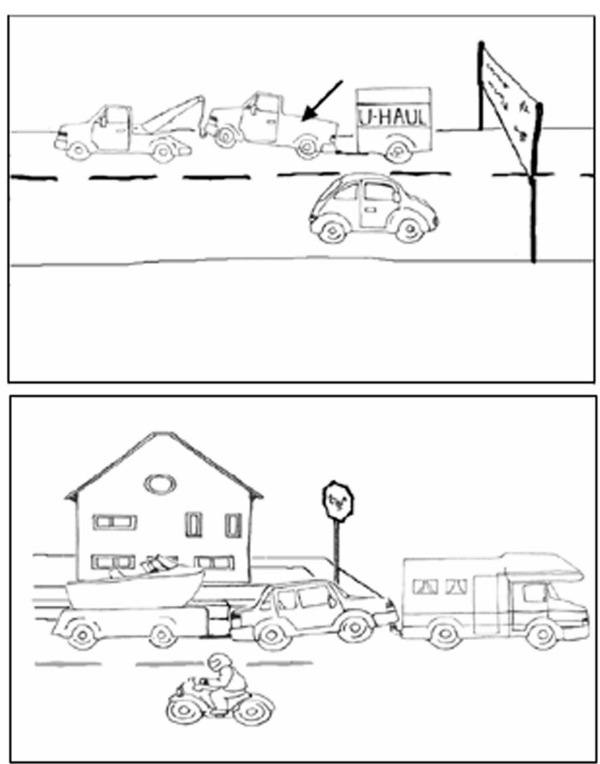

B. Non-living, two-relation, no distractor

D. Living, two-relation, distractor
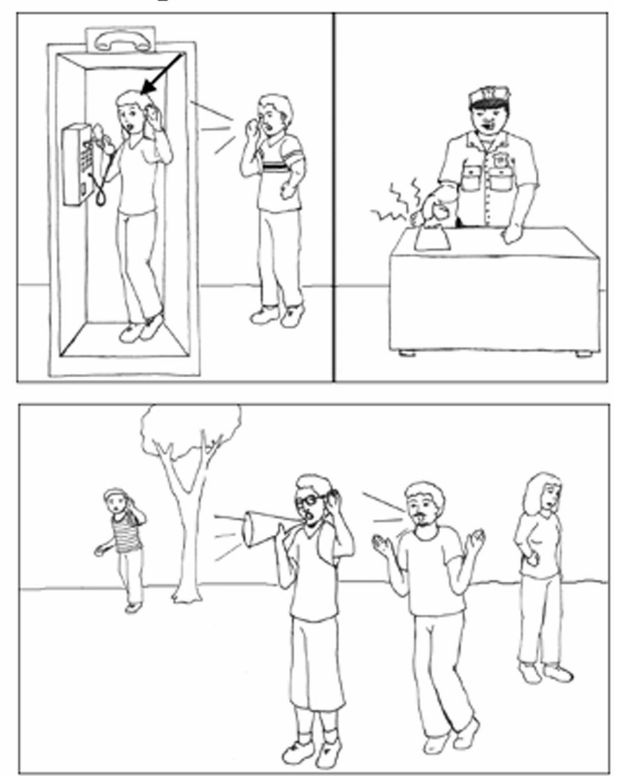

FIGURE 1 | (A) An example is shown of a living, one-relation problem with no distraction. (B) An example of a non-living, two-relation problem with no distraction. (C) A non-living, one-relation problem with a distractor included. (A) A living, two-relation problem with a distractor included. 
et al., 2006) had been designed to investigate the effects of relational complexity and featural distraction on the reasoning abilities of children. It was controlled for knowledge of relations in regard to their youngest age group tested (approximately 3-4 years of age), therefore the relations and objects correspond to simple motion verbs (e.g., chase, fall, pull) and objects commonly encountered by children of that age group including humans, animals, and common household objects.

We modified the task in two ways. First, in the original version of the task, the presentation of the distractor items varied. Some distractor items presented in the target scene were visually identical (e.g., the same object presented in the same orientation) to the key item in the source scene of the analogy. In other problems, the distractor items in the target scene were presented in a different position or orientation compared to that in the source scene. In our modified version, all distractor items were presented in a new position or orientation in the target scene relative to the source assuring that they were never visually identical between source and target scene. Second, we tested for the effect of category of the key items in the analogy (living/non-living), in addition to the original conditions employed by Richland et al. (2006), featural distraction and relational complexity, on reasoning abilities. This was accomplished by dividing the original problem set into examples in which the key items involved in the relations were alive (humans or animals) and examples in which the relational items were non-living. We also added four additional problems in order to achieve an equal set of items for each category. In order to assure that non-living problems were free of living items, we also removed partial images of people in relational roles from problems used in the original picture analogies (Richland et al., 2006) that fit into the non-living category in our modified version.

Each of the 24 scene analogies were divided into two categories. The living category consisted of 12 problems in which the items relevant to the analogy were either people or animals interacting. The non-living category comprised 12 problems that included analogies in which the relevant items were non-living objects that were either manmade such as cars, trains, and lamps, or naturally occurring such as ponds, rocks, and mountains. Within each of these sets, degree of relational complexity was factorially varied. Relational complexity refers to a quantification of the number of relations among elements within the problem that must be considered in order to arrive at a solution. For example, Figure 1A shows a one-relation problem, meaning that this problem can be solved with the single relation "reaching". The boy who is reaching for the cookie jar corresponds to the dog who is reaching for the dog biscuit box. By contrast, Figure 1B shows a two-relation problem in which one must consider the relations "pulling" and "being pulled", as only when both are considered together can one appropriately match the pickup truck in the source scene to the car in the target scene. Other matches are possible on the basis of the pull relation alone (tow truck to camper for the "pulling" relation and trailer to boat for the "being pulled" relation). In the current experiment relational complexity was varied with problems split between one-relation and two-relation scene pairs. We also varied the inclusion or exclusion of a distractor object/character in the target slide that was similar to the item to be mapped in the source slide. The distractor items in the target scenes were similar in that they were the same person, animal, or object as the item with the arrow in the target scene; however, they appeared in a different orientation or position in the target scene. For example, in Figure 1C the water pitcher in the source scene appears again in the target scene in a different position and orientation. Figure $\mathbf{1}$ shows an example of each of the problem types. Four versions of the task were employed. These were counterbalanced such that each of the 24 problems appeared in a one and two-relation format and with and without a distractor across the four versions in order to control for specific item effects.

\section{Design and procedure}

The task was administered to participants on a computer using Eprime 1.2 software (www.pstnet.com/products/e-prime). All participants were given two practice problems that demonstrated the one- and two-relation problems to familiarize them to the task. The following instructions were presented to each participant on the computer screen and read aloud by the experimenter: "You will see two pictures presented one above the other on the screen. Your job is to try and understand what is going on in each of the pictures. There will be a pattern of things happening in the top picture and your job will be to find that same pattern in the bottom picture. You will notice that one thing in the top picture of each set will have a black arrow pointing to it. Your job is to find the object in the bottom picture that best matches the one in the top picture".

Participants indicated their response by pointing to an object in the target picture presented on the computer screen. If the participant answered the sample problem correctly, the researcher would give feedback and then move on to the next sample. If the participant responded incorrectly, the researcher would repeat the description of the relationship shown in the source scene. The experimenter would then ask again which object in the target scene followed the same pattern. The participant was guided until the correct answer was reached with a correct description of the pattern. At this point, the researcher would move on to the next practice problem. If the participant was able to accurately answer the scene analogy, the researcher began the task. If the participant struggled with the second example, the researcher would begin describing the relationship in the top picture, as in the first sample problem. The researcher then moved on to the task.

\section{WORKING MEMORY MEASURES Strategic learning task (SLT)}

This task measures the ability to use working memory to strategically encode high priority information, while avoiding distraction from low priority information. Success depends on effective allocation of attention under three levels of speed demand placed upon working memory. Participants were visually presented with lists of words to recall. Words within each list were printed in one of two colors, with one color designating "high value" words (worth 10 points for accurate recall), and the other color "low value" words (worth 1 point). Participants were instructed to make as high a score as possible. Speed of word presentation was also manipulated by varying stimulus onset-to-onset time, with slow (1500 ms), medium (750 ms), and fast ( $250 \mathrm{~ms}$ ) conditions. Three lists in each speed condition were included for a total of nine trials. To investigate whether there was a cost of the selection process, we included 
two lists of words printed all in black (presented at medium speed) with instructions to simply recall as many words as possible. One pure recall list was presented before the nine color-value lists and the other was presented after the color-value lists. In all cases the dependent measure was number of words recalled. The ratio of high-value to low-value words recalled was calculated to compute a strategic efficiency score, corrected for number of words recalled in each trial. This value ranged from +1 (for perfect strategic efficiency) to -1 for perfect inefficiency, with zero indicating chance performance.

\section{Keep track task (KTT)}

This computerized updating task required adding and deleting items in working memory according to semantic category, thus involving working memory, inhibition, and semantic processing (Yntema and Schulman, 1969; Friedman et al., 2006). On each trial, participants were first presented a set of two, three, or four target categories (e.g., metals, animals, colors), which corresponded to load. Then the subject was presented 15 words, only some of which were exemplars of the target categories, one at a time for $2 \mathrm{~s}$ each. Instructions were to remember only the most recent word presented in each of the target categories. The dependent variables were the number of words correct for Load 2, Load 3, and Load 4.

\section{MAGNETIC RESONANCE IMAGING}

T1-weighted 3D turbo field echo (TFE) axial acquisition series were performed on Philips Intera 3.0T whole body scanners (Philips, Cleveland, OH, USA). Parameters included 1.0-mm thick slices, 0 -mm slice gaps, echo time $(\mathrm{TE})=4.6 \mathrm{~ms} /$ repetition time $(\mathrm{TR})=9.9 \mathrm{~ms}$, field of view $(\mathrm{FOV})=240 \mathrm{~mm}$, and a reconstructed isotropic voxel size of $1.0 \mathrm{~mm}$. Additional parameters included turbo factor $=175$ and flip angle $=8^{\circ}$.

\section{MRI post-processing and FreeSurfer analyses}

Cortical reconstruction and segmentation were performed with the FreeSurfer image analysis suite (Athinoula, 2005). Details of the procedures are described in prior publications (Dale and Sereno, 1993; Dale et al., 1999; Fischl and Dale, 2000; Fischl et al., 1999, 2001, 2002, 2004; Segonne et al., 2004, 2007; Han et al., 2006; Jovicich et al., 2006). Results for each subject were visually inspected to ensure accuracy of registration, skull stripping, segmentation, and cortical surface reconstruction. Manual editing was performed to optimize accuracy of the cortical surfaces. Cortical thickness was measured as the distance between the gray/ white matter boundary and the pial surface at each point on the cortical mantle. Procedures for the measurement of cortical thickness have been validated against histological analysis (Rosas et al., 2002) and manual measurements (Kuperberg et al., 2003; Salat et al., 2004). The data for each participant was re-sampled to an average participant and surface smoothing was performed, using a 10-mm full-width half-maximum Gaussian kernel, prior to statistical analysis.

Once the processing stream and manual editing were satisfactorily completed, the FreeSurfer query design estimate contrast (QDEC) application was used to fit a general linear model at each surface vertex for the relation of cortical thickness to the cognitive performance variables. Statistical parametric maps of the entire cortical mantle were generated to show the relation of cortical thickness across the brain surface to cognitive performance variables. Due to the exploratory nature of the analysis, a statistical threshold of $p<0.01$ was used for display purposes, in order to show the spatial extent of the areas involved.

\section{STATISTICS}

Repeated measures ANOVAs were conducted on the four analogy conditions (group, presence of distractor, relational level, animacy). Age was also entered into the model in order to control for age effect and to see whether it modifies the group differences on the outcomes.

The SLT generated measures of overall word recall for the two non-strategic recall conditions (i.e., pre- and post-strategic memory trials). Word recall means were also generated for the strategic conditions (i.e., slow, medium, and fast). In addition, mean recall efficiency scores were also calculated for the three strategic conditions. This resulted in five recall scores and three efficiency scores. These eight scores were also tested for correlation with mean proportion correct for each of six problem categories from the picture analogy task (one-relation problems, two-relation problems, living problems, non-living problems, distractor, and non-distractor problems) using Spearman correlation coefficients separately for each participant group.

The KTT produced one percentage correct score for each of the performance conditions (i.e., two, three, or four category conditions). These scores were tested for correlation with the six picture analogy problem type means using Spearman correlation coefficients for each participant group separately.

\section{RESULTS}

\section{PICTURE ANALOGY TASK}

Typically developing youth were significantly better at reasoning analogous roles among elements in scenes than were youth with TBI, $F(1,17)=10.55, p=0.0047$ (refer to Figure 2). Age had

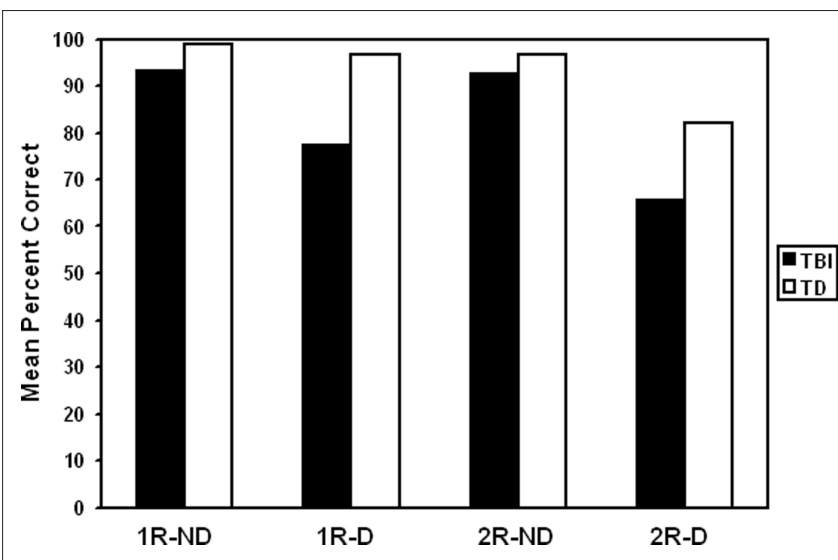

FIGURE 2 |TD participants showed greater overall performance than TBI participants across the task. The number of relations also had a significant effect on performance, with two-relation analogies (2R) resulting in lower performance than single-relation analogies (1R) for both groups. Presence of distractor $(D)$ interacted with relation level so that the effect of relation level was more pronounced with distractors than without distractors (ND). 
an effect that depended on group, $F(1,17)=5.58, p=0.0303$, such that younger adolescents with TBI performed more poorly than older adolescents, but this age difference was not apparent in TD youth.

\section{Presence of distractor}

Presence of distractor had an effect on performance, $F(1,17)=17.87$, $p=0.0006$, with problems that included a distractor being solved at a lower rate than those without distractors. Distractor presence interacted with participant group, $F(1,17)=4.54, p=0.0481$, such that youth with TBI were more negatively affected by distractors than were TD youth. Presence of distractor also interacted with relational level, so that the effect of relational level was more pronounced with distractors than without, $F(1,17)=6.23, p=0.0231$ (refer to Figure 2). No other effects were significant involving distractor presence.

\section{Relation level}

The number of relations had a significant effect on performance in both groups, with two-relation analogies showing more difficulty than single-relation analogies, $F(1,17)=12.07, p=0.0029$ (refer to Figure 2). There were no significant interactions between relation level and any other variable.

\section{Problem type: living versus non-living}

There were no significant differences between the living and nonliving problem types, $F(1,17)=0.00, p=0.9747$.

\section{RELATIONSHIP BETWEEN ANALOGY TASK AND WORKING MEMORY SLT}

Correlational analyses revealed several significant correlations between SLT category scores and each problem type from the picture analogy task. The TBI group showed significant correlations on the following pairs: non-living problems and recall at slow speed ( rho $=.86, p<0.01)$, distractor problems and recall at slow speed (rho $=0.83, p=0.01$ ), non-living problems and recall at medium speed (rho $=0.96, p<0.001)$, and distractor problems and recall at medium speed ( $r h o=0.77, p<0.05$ ). For the TBI participants, there were also strong correlations between SLT efficiency at the medium presentation speed and the following analogy task categories: one-relation problems $($ rho $=0.87$, $p<0.01$ ), two-relation problems ( $r$ ho $=0.88, p<0.01$ ), living problems $(\mathrm{rho}=0.87, p<0.01)$, distractor problems $(\mathrm{rho}=0.80$, $p<0.05$ ), and non-distractor problems ( $\mathrm{rho}=0.83, p=0.01$ ). In the TD participants, the only significant correlation was SLT efficiency at the medium presentation and distractor problem performance ( $r$ ro $=0.69, p<0.05$ ). Refer to Table 1 for a full report of these correlation values.

\section{KTT}

Correlational analyses revealed that two-category performance was significantly correlated with mean performance on living problems from the picture analogy task for TBI participants ( rho $=0.93$, $p<0.01$ ). There were no other significant correlations present in either participant group. Table 2 provides a complete report of these correlation values. Given the small sample size in this study, we computed effect sizes. Both categories of one-relation problems showed large effect sizes (Cohen's $d=0.77-1.40$ ), as did problems containing two-relation with distractor (Cohen's $d=1.55$ ). Tworelation problems without distractor showed a medium effect size (Cohen's $d=0.39$ ).

\section{RELATIONSHIP BETWEEN ANALOGY TASK AND CORTICAL THICKNESS}

Correlational relationships were measured by correlating cortical gray matter thickness with task performance using a general linear model. The strongest correlations were found for the TD participants for overall task performance. Figure 3 shows regions of the brain showing an inversely correlated relationship between overall analogy accuracy and cortical thickness. Notably, the anterior PFC (left BA10), bilateral anterior and posterior lateral PFC, bilateral superior and inferior temporal gyri, and the medial PFC showed these correlations in the TD group. There was less evidence of a clear correlational relationship in the TBI group and performance overall, but some inverse correlations were observed in the left medial orbitofrontal cortex and the left superior frontal gyrus. Similar relationships in similar areas were observed for accuracy on all two-relation analogies and gray matter thickness in the TD group (see Figure 4). Accuracy on all problems with distractors present showed an inverse correlational relationship with cortical thickness with foci in the left superior temporal gyrus, left middle temporal gyrus, right inferior frontal gyrus, left posterior cingulate for the TD group. Additional foci observed in the TBI group included the anterior left dorsal PFC and right orbitofrontal cortex (refer to Figure 4). Overall, the cortical thickness data showed the strongest correlational relationships between thinner cortex and superior performance overall and at the higher relation level in TD adolescents. This is broadly consistent with cortical maturity, or efficiency in anterior PFC and temporal lobe regions.

\section{DISCUSSION}

Adolescents with TBI show predictable reasoning deficits. They exhibit lower overall performance, but particularly in cases in which distraction is present, and when relational complexity is higher coupled with distraction. This is consistent with an overall deficit in working memory maintenance. TBI participants showed deficits consistent with a lowered ability to screen out irrelevant, but visually competing representations. Lastly, TBI individuals were insensitive to the manipulation of problems that involved living compared to non-living items. The TD group performed at relatively high levels across all problem types, and there was not an age difference present in this group of 13- to 19-year olds. This is consistent with prior results demonstrating that in a similar set of scene analogies, adolescents achieve high competence levels by ages 13-14 years (Richland et al., 2006). This was not the case for TBI subjects who did show an age effect, suggesting that younger individuals who sustain a TBI are particularly impaired. This is consistent with the executive function literature in TBI, which indicates that age of injury can have an influence on higher cognitive abilities, with injuries in younger individuals leading to greater higher cognition deficits (Andersen et al., 1999; Ewing-Cobbs et al., 2004; Anderson and Catroppa, 2005). Fortunately, there is also evidence that younger individuals with TBI impairments may have a greater potential for recovery in certain areas including inhibitory control (Leblanc et al., 2006). 
Table 2 | Correlations between performance on the analogy task and the working memory tasks.

\begin{tabular}{|c|c|c|c|c|c|c|c|c|c|c|c|}
\hline & $\begin{array}{l}\text { Overall } \\
\text { recall I }\end{array}$ & $\begin{array}{l}\text { Overall } \\
\text { recall II }\end{array}$ & $\begin{array}{l}\text { Recall } \\
\text { slow } \\
\text { pace }\end{array}$ & $\begin{array}{l}\text { Recall } \\
\text { medium } \\
\text { pace }\end{array}$ & $\begin{array}{l}\text { Recall } \\
\text { fast } \\
\text { pace }\end{array}$ & $\begin{array}{l}\text { Efficiency } \\
\text { slow } \\
\text { pace }\end{array}$ & $\begin{array}{l}\text { Efficiency } \\
\text { medium } \\
\text { pace }\end{array}$ & $\begin{array}{l}\text { Efficiency } \\
\text { fast pace }\end{array}$ & $\begin{array}{l}\text { КTT: } 2 \\
\text { category }\end{array}$ & $\begin{array}{l}\text { KTT: } 3 \\
\text { category }\end{array}$ & $\begin{array}{l}\text { КTT: } 4 \\
\text { category }\end{array}$ \\
\hline \multicolumn{12}{|c|}{ One-relation } \\
\hline$\rho$ & 0.650 & -0.200 & 0.600 & 0.625 & 0.346 & 0.350 & $0.871 * *$ & 0.405 & 0.125 & -0.1850 & -0.062 \\
\hline \multicolumn{12}{|c|}{ Two-relation } \\
\hline$\rho$ & 0.453 & -0.356 & 0.704 & 0.528 & 0.000 & 0.453 & $0.877^{* *}$ & 0.235 & 0.509 & 0.000 & 0.204 \\
\hline$p$ & 0.259 & 0.386 & 0.051 & 0.178 & 1.000 & 0.260 & 0.004 & 0.576 & 0.303 & 1.000 & 0.699 \\
\hline$n$ & 8 & 8 & 8 & 8 & 8 & 8 & 8 & 8 & 6 & 6 & 6 \\
\hline \multicolumn{12}{|c|}{ Living } \\
\hline \multicolumn{12}{|c|}{ Non-living } \\
\hline$\rho$ & 0.646 & 0.292 & $0.857^{* *}$ & $0.957^{* *}$ & 0.362 & 0.075 & 0.488 & -0.037 & -0.470 & 0.194 & 0.164 \\
\hline$p$ & 0.083 & 0.483 & 0.007 & 0.000 & 0.378 & 0.861 & 0.220 & 0.932 & 0.347 & 0.713 & 0.756 \\
\hline$n$ & 8 & 8 & 8 & 8 & 8 & 8 & 8 & 8 & 6 & 6 & 6 \\
\hline \multicolumn{12}{|c|}{ Distraction } \\
\hline$\rho$ & 0.553 & -0.114 & $0.830 *$ & $0.767^{*}$ & 0.416 & 0.340 & $0.803^{*}$ & 0.148 & 0.136 & 0.358 & 0.315 \\
\hline$p$ & 0.155 & 0.788 & 0.011 & 0.026 & 0.305 & 0.411 & 0.017 & 0.726 & 0.797 & 0.486 & 0.545 \\
\hline$n$ & 8 & 8 & 8 & 8 & 8 & 8 & 8 & 8 & 6 & 6 & 6 \\
\hline \multicolumn{12}{|c|}{ No-Distraction } \\
\hline$\rho$ & 0.254 & $-0.723^{*}$ & 0.296 & 0.127 & -0.334 & 0.592 & $0.830^{*}$ & 0.456 & 0.801 & -0.257 & -0.103 \\
\hline$p$ & 0.545 & 0.043 & 0.477 & 0.765 & 0.419 & 0.122 & 0.011 & 0.256 & 0.056 & 0.623 & 0.846 \\
\hline \multicolumn{12}{|c|}{ Two-relation } \\
\hline$\rho$ & -0.336 & 0.000 & -0.162 & -0.026 & 0.285 & 0.200 & 0.528 & 0.219 & 0.000 & 0.481 & -0.275 \\
\hline$p$ & 0.377 & 1.000 & 0.677 & 0.948 & 0.458 & 0.606 & 0.144 & 0.571 & 1.000 & 0.275 & 0.551 \\
\hline$n$ & 9 & 9 & 9 & 9 & 9 & 9 & 9 & 9 & 7 & 7 & 7 \\
\hline \multicolumn{12}{|c|}{ Living } \\
\hline$\rho$ & 0.140 & 0.000 & 0.000 & -0.211 & 0.560 & 0.550 & 0.411 & 0.274 & -0.167 & 0.629 & 0.105 \\
\hline$p$ & 0.720 & 1.000 & 1.000 & 0.586 & 0.117 & 0.125 & 0.272 & 0.476 & 0.721 & 0.130 & 0.823 \\
\hline$n$ & 9 & 9 & 9 & 9 & 9 & 9 & 9 & 9 & 7 & 7 & 7 \\
\hline \multicolumn{12}{|c|}{ Non-living } \\
\hline$\rho$ & -0.363 & 0.000 & -0.394 & -0.298 & 0.000 & -0.032 & 0.323 & -0.061 & -0.114 & 0.113 & -0.563 \\
\hline$p$ & 0.338 & 1.000 & 0.294 & 0.436 & 1.000 & 0.934 & 0.397 & 0.869 & 0.808 & 0.810 & 0.188 \\
\hline$n$ & 9 & 9 & 9 & 9 & 9 & 9 & 9 & 9 & 7 & 7 & 7 \\
\hline \multicolumn{12}{|c|}{ Distraction } \\
\hline$\rho$ & -0.095 & 0.228 & -0.152 & -0.239 & 0.152 & 0.225 & $0.690^{*}$ & 0.112 & 0.114 & 0.184 & -0.072 \\
\hline$p$ & 0.808 & 0.554 & 0.697 & 0.536 & 0.696 & 0.561 & 0.040 & 0.775 & 0.808 & 0.692 & 0.879 \\
\hline$n$ & 9 & 9 & 9 & 9 & 9 & 9 & 9 & 9 & 7 & 7 & 7 \\
\hline \multicolumn{12}{|c|}{ No-distraction } \\
\hline$\rho$ & -0.326 & -0.280 & -0.371 & -0.234 & 0.093 & -0.046 & -0.183 & -0.091 & 0.354 & 0.297 & -0.594 \\
\hline$p$ & 0.391 & 0.466 & 0.325 & 0.544 & 0.811 & 0.907 & 0.683 & 0.815 & 0.437 & 0.518 & 0.160 \\
\hline$n$ & 9 & 9 & 9 & 9 & 9 & 9 & 9 & 9 & 7 & 7 & 7 \\
\hline
\end{tabular}

${ }^{*} p<0.05 ;{ }^{*} p<0.01$. 
TD
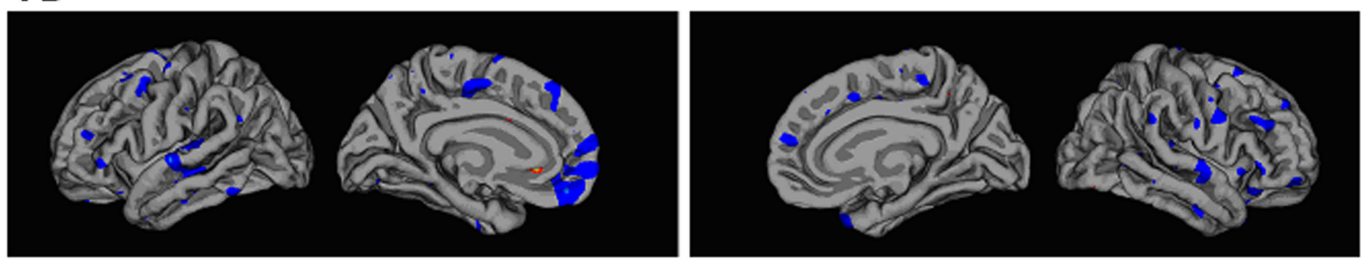

TBI
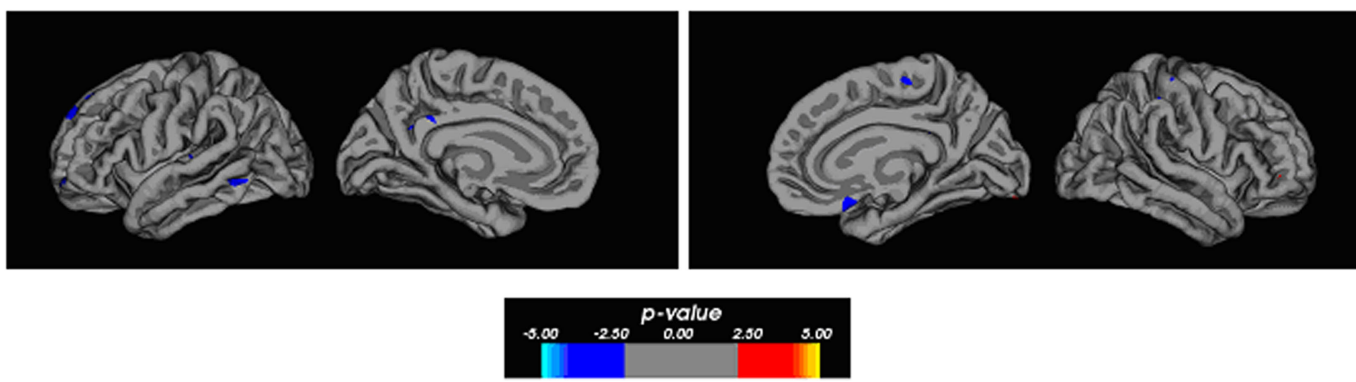

FIGURE 3 | Correlation maps showing significant correlational relationships between cortical thickness and overall accuracy on the analogy task. Regions shown in blue show an inverse correlation such that thinner cortex is associated with higher performance. The TD participants showed significant inverse correlations within several lateral areas within the frontal and temporal lobes. The frontal regions extend between the ventral and dorsal portions with a particularly strong focus within the medial frontal pole. The TBI participants show less of a strong correlational relationship on this measure, which may indicate that their performance is affected by the lack of developmentally typical changes in the cortical mantle within the frontal and temporal lobes. The $p$-value in the color bar refers to a - $\log (10) p$-value for significance level as is customary for FreeSurfer software.
A relational complexity effect was found for both groups indicating that two-relation problems were more difficult than one-relation problems. This is consistent with our predictions and similar to the reports of other studies of analogy and relational reasoning (Richland et al., 2006; Krawczyk et al., 2008). While the relational complexity had an impact on accuracy, both groups were still at around ninety percent accuracy on two-relation problems collapsed across animacy conditions, but without distraction (refer to Figure 2). The effects of the two relations on problems became clearer when crossed with distraction. The distraction by relation level interaction indicates that it is the combination of increased complexity and distraction which reveals reasoning deficits in TBI individuals. In the case of TD participants, performance was high for all problems that had no distraction, while it was lower for the distractor problems. TBI participants showed reductions in performance whenever distraction was present, but this was most acute in the case of all two-relation problems involving distraction. Overall, TBI participants were less effective in performing higher complexity problems and particularly those with distraction present. Similar findings have been observed previously with patients with frontal dementia (Waltz et al., 1999; Morrison et al., 2004; Krawczyk et al., 2008) and Alzheimer's disease (Waltz et al., 2004).

Both participant groups exhibited lower performance when distracting items were present, but these items had a disproportionate effect on TBI participants relative to the TD group. This result also supports an executive control deficit in TBI and specifically a deficit of inhibitory control over distractors, as has been observed in the analogy performance of frontal dementia patients (Morrison et al., 2004; Krawczyk et al., 2008). The ability to screen distractors has been shown to improve with age between young childhood and early ado- lescence (Richland et al., 2006), but our data indicate that TBI may lead to reductions in performance that reduce the capacity of adolescents sustaining TBI relative to their age matched peers. This may be particularly the case for younger individuals with TBI (age 12-14), as there was an overall age by group interaction demonstrating that younger TBI individuals perform worse than older individuals.

The lack of an effect of animacy on reasoning performance may have been due to our rather broad manipulation. We noted that most individuals in both groups showed a slight advantage for living over non-living item analogies in most cases. This may be due to the fact that interactions among living items tended to involve motion, while non-living items were mostly stationary, thus the salience of the relations may not have been as high with the nonliving problems. As prior studies have reported deficits in social cognition that are associated with TBI adolescents (Hanten et al., 2008; Levin et al., 2009) this appears to be an important factor that may be demonstrated to influence reasoning in TBI. In future work, we will attempt to manipulate this factor with greater sensitivity and on multiple social dimensions in order to determine its influence on reasoning performance.

Working memory measures also indicated that TBI adolescents have impairments that may underlie the deficits observed in analogical reasoning. Specifically, TBI adolescents have greater difficulty with updating working memory and screening distraction. Both of these tasks relied upon properly allocating attention toward incoming stimuli and making efficient judgments about which items to encode and which items to discard. Such deficits in core attention and memory abilities could serve as a basis for the difficulties that TBI adolescents encountered when faced with more complex two-relation reasoning problems. 


\section{A TD}
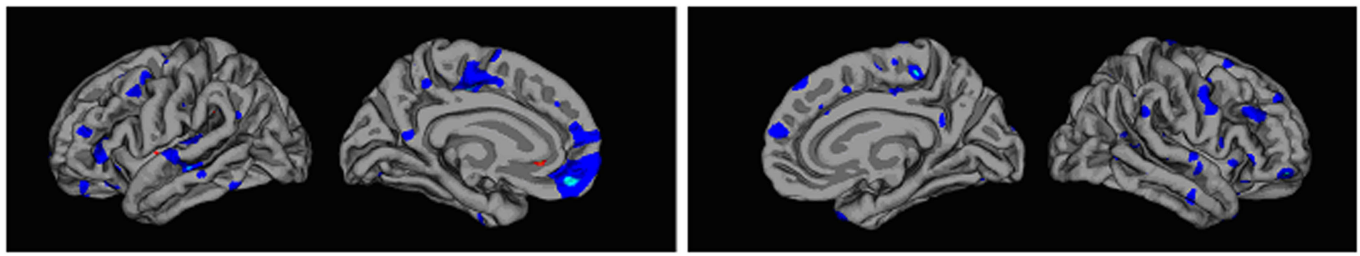

TBI
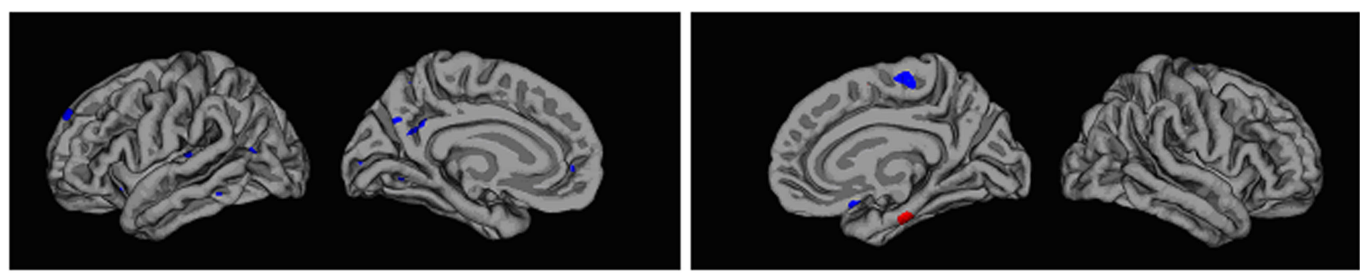

B TD
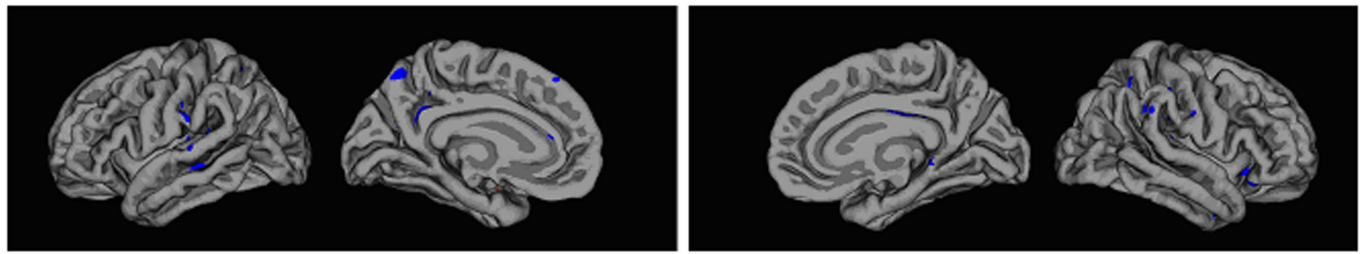

TBI
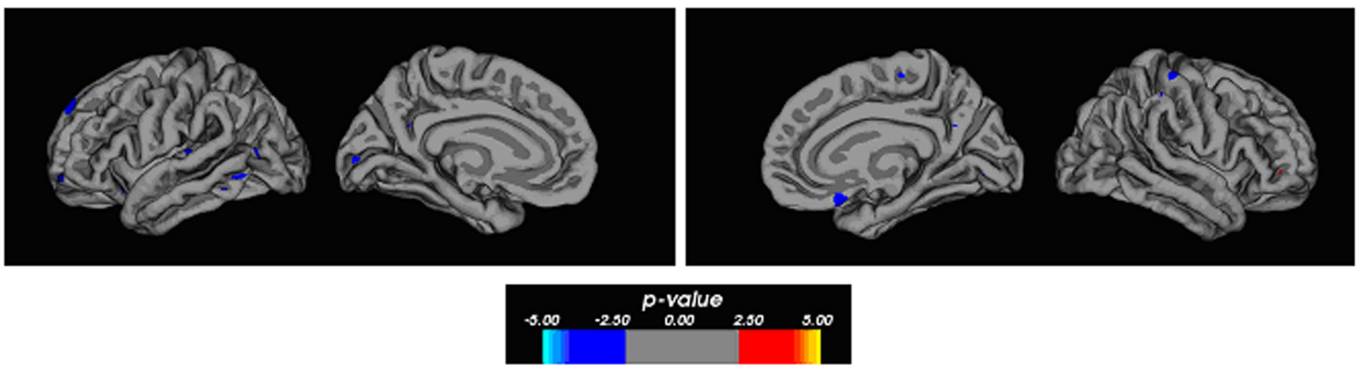

FIGURE 4 | Correlation maps between gray matter thickness and performance. (A) The maps show evidence of strong inverse correlational relationships between two-relation performance and thickness in several cortical areas within the TD participants. The foci of correlations include areas of the dorsal and ventral frontal lobes with considerable area within the left anterior PFC. Additional areas of correlation included the middle cingulate cortex, and temporal lobe regions. Few regions showed strong correlations within the TBI participants on this same comparison. (B) Both TD and TBI participants showed some degree of inverse correlation between cortical thickness and performance on distractor problems. These foci were primarily observed in the temporal lobes and medial parietal cortex, and medial-dorsal PFC. The $p$-value in the color bar refers to $a-\log (10) p$-value that is standard in analyses performed in FreeSurfer.
Our correlational analyses suggest that the TBI group showed strong relationships between certain memory conditions and analogy problem type conditions. The recall rate on the SLT varied significantly with two of the more difficult problem types in the analogy task (distractor problems and non-living problems). Correlations were observed at the slow and medium presentation rates with these analogy variables. The fast pacing condition of the task failed to show strong correlations, perhaps due to the overall difficulty imposed upon this group at the higher rates of speed. It is likely that TD youth failed to show robust correlations due to their high overall level performance on both of the tasks leading to a reduction in variability. For the TBI group these correlations likely reflect analogical performance being mediated by working memory ability. The large number of correlations between the SLT efficiency scores at medium speed and nearly all categories of the analogy task further link strategic allocation of attention to successful analogy performance. Lastly, the correlation between the KTT task and living problems for the TBI group supports a similar linkage.

Our gray matter imaging results were consistent with prior functional studies of analogical reasoning performed with younger children (Wright et al., 2007) and adults (Bunge et al., 2005; Green et al., 2006). Convergent with functional imaging studies, we demonstrated significant behavior-cortical thickness relationships within the frontal pole, PFC, and temporal cortex. These results were most robust for TD adolescents and showed an inverse correlation, with decreased cortical thickness associated with stronger analogy performance in TD participants. This finding is consistent with increased cortical maturity, through apoptosis and increases 
in myelination (Sowell et al., 1999; Shaw et al., 2008; Giedd et al., 2009), supporting the functions relevant to analogical reasoning. The PFC involvement is likely due to the need for executive control in processing analogical over feature distractor-based matches (Geake and Hansen, 2010). A similar relationship has been reported between gray matter thickness and performance on fluid reasoning tasks in childhood and adolescence (O'Hare et al., 2009). The involvement of the temporal lobes is consistent with the need for semantic memory representations relevant to analogical reasoning (Morrison et al., 2004; Krawczyk et al., 2008). Our results are consistent with performance increases in development being associated with overall cortical maturity within the PFC, temporal, and parietal lobes. It should be noted that in individual differences in general intelligence tasks in adulthood tend to show a different relationship with cortical thickness often correlating with higher performance in the PFC (Haier et al., 2004; Colom et al., 2006). Thus, after maturation, there may be different patterns observed in some fluid reasoning tasks. Our TBI participants showed weaker task-cortical thickness correlations consistent with gray matter disruption associated with brain injuries. Possible mechanisms for this lack of relation include altered apoptosis or plasticity changes that obscure the maturity-totask relationships that may otherwise be observed.

There are certain limitations in the present study that may be addressed in future research on this topic. Notably, the sample size in our population was relatively small. This may have affected our ability to observe certain effects. For instance the animacy manipulation did not reveal significant differences, though there is evidence from other studies that social cognition is affected by TBI (Janusz et al., 2002; Yeates et al., 2002; Hanten et al., 2008). The lack of an effect for the living and non-living aspect of the analogy problems may be shown with a larger sample size. Since participants performed at a relatively high level across the analogy sets, it is also possible that ceiling effects limited our ability to detect differences based on the living/non-living manipulation. This was an exploratory manipulation and there was little prior literature to guide our adjustments to the stimuli, so it is also possible that we simply did not employ a measure sensitive enough to capture effects that may have reflected social cognition in these TBI adolescents. Lastly,

\section{REFERENCES}

Andersen, S. W., Bechara, A., Damasio, H., Tranel, D., and Damasio, A. R. (1999). Impairment of social and moral behavior related to early damage in human prefrontal cortex. Nat. Neurosci. 2, 1032-1037.

Anderson, V., and Catroppa, C. (2005). Recovery of executive skills following paediatric traumatic brain injury (TBI): A 2 year follow-up. Brain Inj. 19, 459-470.

Athinoula, A. (2005). Martinos Center for Biomedical Imaging. Freesurfer.

Berryhill, P., Lilly, M., Levin, H.S., Hillman, G. R., Mendelsohn, D., Brunder, D. G., Fletcher, J. M., Kufera, J., Kent, T. A., Yeakley, J., Bruce, D., Eisenberg, H. M., McComb, J. G., and Rekathe, H. L. (1995). Frontal lobe changes after severe diffuse closed head injury in

there is potential that the TBI adolescent sample may have had individual variability that limits its generalizability to a broader TBI population. This is always a concern when dealing with TBI, given that head trauma produces lesions and white matter degeneration that varies in extent and location on an individual basis due to the precise characteristics of the trauma.

In conclusion, individuals with TBI show deficits in analogical reasoning that appear to be related to loss of executive function abilities due to brain injury. The reductions in cortical thickness characteristic of MSTBI cases are likely sources of frontal systemrelevant injuries that result in less adequate resources for problem solving. Specifically, TBI adolescents have more difficulty than healthy peers in solving analogy problems when feature match distractors are present. The possibility of feature matches may lead to either impulsive choices in which matches are based on perceptual similarity, or choices that are based on a reduced ability to process and perceive the broader overall meaning of the relational interactions within a scene. TBI adolescents' difficulties with analogical reasoning are most clear when relational complexity of a scene is high and distraction is present. TBI adolescents also fail to show robust cortical volume relationships to task performance as observed in healthy adolescents within the frontal and temporal lobes. A goal for future research will be gaining a better understanding how TBI rehabilitation can improve the executive control functions necessary for reasoning in this population.

\section{ACKNOWLEDGMENTS}

We thank Lindsey Richland for assistance with the task materials and procedure. We thank Carrie McAdams, Kevin Murch, Ehsan Shokri, and Gloria Yang for helpful comments and suggestions. We also acknowledge the assistance of Drs. Jill V. Hunter and Zili Chu in image acquisition and pre-processing. This work was supported by NIH grant NS-21889 (Harvey S. Levin). The content is solely the responsibility of the authors and does not necessarily represent the official views of the National Institutes of Health. We would also like to acknowledge the generous support by Mission Connect of the TIRR Foundation.

meets a stringent test of the relational integration hypothesis. Neuroimage. 46, 338-342.

Bunge, S. A., Wendelken, C., Badre, D., Wagner, A. D. (2005). Analogical reasoning and prefrontal cortex: evidence for separable retrieval and integration mechanisms. Cereb Cortex. 15, 239-249.

Christoff, K., Keramatian, K., Alan, G. M., Smith, R., and Maedler, B. (2009). Representational topography in lateral prefrontal cortex according to levels of abstraction. Brain Res. 1286, 94-105.

Colom, R., Jung, R. E., and Haier, R. J. (2006). Distributed brain sites for the g-factor of intelligence. Neuroimage 31, 1359-1365.

Crone, E. A., Wendelken, C., van Leijenhorst, L., Honomichl, R. D.,
Christoff, K., and Bunge, S. A. (2009). Neurocognitive development of relational reasoning. Dev. Sci. 12, 55-66.

Dale, A. M., Fischl, B., and Sereno, M. I. (1999). Cortical surface-based analysis. I. Segmentation and surface reconstruction. Neuroimage 9, 179-194.

Dale, A. M., and Sereno, M. I. (1993). Improved localization of cortical activity by combining EEG and MEG with MRI cortical surface reconstruction: a linear approach. J. Cogn. Neurosci. 5, 162-176.

Dennis, M., Guger, S., Roncadin, C., Barnes, M., and Schachar, R. (2001). Attentional-inhibitory control and social-behavioral regulation after childhood closed head injury: do biological, developmental, and recovery variables predict outcome? J. Int. Neuropsychol. Soc. 7, 683-692. 
Ewing-Cobbs, L., Prasad, M. R., Landry, S. H., Kramer, L., and DeLeon, R. (2004). Executive functions following traumatic brain injury in young children: a preliminary analysis. Dev. Neuropsychol. 26, 487-512.

Fischl, B., and Dale, A. M. (2000). Measuring the thickness of the human cerebral cortex from magnetic resonance images. Proc. Natl. Acad. Sci. 97 , 11050-11055.

Fischl, B., Liu, A., and Dale, A. M. (2001). Automated manifold surgery: constructing geometrically accurate and topologically correct models of the human cerebral cortex. IEEE Trans. Med. Imaging, 20, 70-80.

Fischl, B., Salat, D. H., Busa, E., Albert, M., Dieterich, M., Haselgrove, J.C., van der Kouwe, A. J., Killiany, R. J., Kennedy, D., Klaveness, S., Montillo, A., Makris, N., Rosen, B., and Dale, A. M. (2002). Whole brain segmentation: automated labeling of neuroanatomical structures in the human brain. Neuron 33, 341-355.

Fischl, B., Salat, D. H., van der Kouwe, A. J., Makris, N., Segonne, F., Quinn, B. T., and Dale, A. M. (2004). Sequenceindependent segmentation of magnetic resonance images. Neuroimage 23, S69-S84.

Fischl, B., Sereno, M. I., Tootell, R. B., and Dale, A. M. (1999). High-resolution intersubject averaging and a coordinate system for the cortical surface. Human Brain Mapp. 8, 272-284.

Friedman, N. P., Miyake, A., Corley, R. P., Young, S. E., Defries, J. C., and Hewitt, J. K. (2006). Not all executive functions are related to intelligence. Psychol. Sci. $17,172-179$.

Geake, J. G., and Hansen, P. C. (2010). Functional neural correlates of fluid and crystallized analogizing. Neuroimage 49, 3489-3497.

Giedd, J. N., Lalonde, F. M., Celano, M. J., White, S. L., Wallace, G. L., Lee, N. R., and Lenroot, R. K. (2009). Anatomical brain magnetic resonance imaging of typically developing children and adolescents. J. Am. Acad. Child Adolesc. Psychiatry 48, 465-470.

Gentner, D. (1983). Structure-mapping: a theoretical framework for analogy. Cogn. Sci.: A Multidiscip. J. 7, 155-170.

Gentner,D., and Rattermann, M.J. (1991). "Language and the career of similarity," in Perspectives on Language and Thought:Interrelationsin Development, eds S. A. Gelman and J. P. Byrnesm (New York, NY: Cambridge University Press), 225-277.

Gentner, D., and Toupin, C. (1986). Systematicity and surface similarity in the development of analogy. Cogn. Sci. A Multidiscip. J. 10, 277-300.
Ghosh, A., Wilde, E. A., Hunter, J. V., Bigler, E. D., Chu, Z., Li, X., Vasquez, A. C., Menefee, D., Yallampalli, R., and Levin, H. S. (2009). The relation between Glasgow coma scale score and later cerebral atrophy in pediatric traumatic brain injury. Brain Inj. 23: 228-233.

Goswami, U. (2001). Cognitive development: no stages please - we're British. Br. J. Psychol. 92, 257-277.

Goswami, U., and Brown, A. L. (1989). Melting chocolate and melting snowmen: Analogical reasoning and causal relations. Cognition 35, 69-95.

Goswami, U., Leevers, H., Pressley, S., and Wheelwright, S. (1998). Causal reasoning about pairs of relations and analogical reasoning in young children. Br. J. Dev. Psychol. 16, 553-569.

Green, A. E., Fugelsang, J. A., Kraemer, D. J., Shamosh, N. A., and Dunbar, K. N. (2006). Frontopolar cortex mediates abstract integration in analogy. Brain Res. 1096, 125-137.

Green,A.E., Kraemer, D. J., Fugelsang, J.A., Gray, J. R., and Dunbar, K. N. (2010). Connecting Long Distance: Semantic Distance in Analogical Reasoning Modulates Frontopolar Cortex Activity. Cereb Cortex 20, 70-76.

Haier, R. J., Jung, R. E., Yeo, R. A., Head, K., and Alkire, M. T. (2004). Structural brain variation and general intelligence. Neuroimage 23, 425-433.

Halford, G. S., Maybery, M. T., O'Hare, A. W., and Grant, P. (1994). The development of memory and processing capacity. Child Dev. 65, 1338-1356.

Han, X., Jovicich, J., Salat, D. H., van der Kouwe, A. J., Quinn, B. T., Czanner, S., Busa, E., Pacheco, J., Albert, M., Killiany, R. J., Maguire, P., Rosas, D., Makris, N., Dale, A. M., Dickerson, B. C., and Fischl, B. (2006). Reliability of MRI-derived measurements of human cerebral cortical thickness: the effects of field strength, scanner upgrade and manufacturer. Neuroimage 32 , 180-194.

Hanten, G., Wilde, E. A., Menefee, D. S., Li, X., Lane, S., Vasquez, C., Chu, Z., Ramos, M. A., Yallampalli, R., Swank, P., Chapman, S. B., Gamino, J., Hunter, J.V., and Levin, H.S. (2008). Correlates of social problem solving during the first year after traumatic brain injury in children. Neuropsychology 22, 357-370.

Hiscock, M., Inch, R., and Gleason, A. (2002). Raven's progressive matrices performance in adults with traumatic brain injury. Appl. Neuropsychol. 9, 129-138.

Holyoak, K. J., and Thagard, P. (1996). Mental Leaps: Analogy in Creative Thought. Cambridge, MA: MIT Press.
Hummel, J. E., and Holyoak, K. J. (1997). Distributed representations of structure: a theory of analogical access and mapping. Psychol. Rev. 104, 427-466.

Hummel, J. E., and Holyoak, K. J. (2003). A symbolic-connectionist theory of relational inference and generalization. Psychol. Rev. 110, 220-264.

Janusz, J. A., Kirkwood, M. W., Yeates, K. O., and Taylor, H. G. (2002). Social problem-solving skills in children with traumatic brain injury: long-term outcomes and prediction of social competence. Child Neuropsychol. 8, 179-194.

Jovicich, J., Czanner, S., Greve, D., Haley, E., van der Kouwe, A. J., Gollub, R., Kennedy, D., Schmitt, F., Brown, G. Macfall, J., Fischl, B., and Dale, A. M. (2006). Reliability in multi-site structural MRI studies: effects of gradient non-linearity correction on phantom and human data. Neuroimage $30,436-443$.

Konrad, K., Gauggel, S., Manz, A., and Scholl, M. (2000a). Inhibitory control in children with traumatic brain injury (TBI) and children with attention deficit/hyperactivity disorder (ADHD). Brain Inj. 14, 859-875.

Konrad, K., Gauggel, S., Manz, A., and Scholl, M. (2000b). Lack of inhibition: a motivational deficit in children with attention deficit/hyperactivity disorder and children with traumatic brain injury. Child Neuropsychol. 6, 286-296.

Kotovsky, L., and Gentner, D. (1996). Comparison and categorization in the development of relational similarity. Child Dev. 67, 2797-2822.

Krawczyk, D. C., Morrison, R. G., Viskontas, I., Holyoak, K. J., Chow, T. W., Mendez, M. F., Miller, B. L., and Knowlton, B. J. (2008). Distraction during relational reasoning: the role of prefrontal cortex in interference control. Neuropsychologia 46 2020-2032.

Kuperberg, G. R., Broome,M.R., McGuire, P. K., David, A. S., Eddy, M., Ozawa, F., Goff, D., West, W. C., Williams, S. C., van der Kouwe, A. J., Salat, D. H., Dale, A. M., and Fischl, B. (2003). Regionally localized thinning of the cerebral cortex in schizophrenia. Arch. Gen. Psychiatry 60, 878-888.

Langlois, J. A., Kegler, S. R., Butler, J. A., Gotsch, K. E., Johnson, R. L., Reichard, A. A., Webb, K. W., Coronado, V. G., Selassie, A. W., and Thurman, D. J. (2003). Traumatic brain injury-related hospital discharges. MMWR Surveill. Summ. 52, 1-20.

Langlois, J. A., Rutland-Brown, W., and Thomas, K. E. (2005). The incidence of traumatic brain injury among children in the United States: differences by race. J. Head Trauma Rehabil. 20 , 229-238.
Leblanc, N., Chen, S., Swank, P. R., Levin, H., and Schachar, R. (2006) Impairment and recovery in inhibitory control after traumatic brain injury in children: effect of age at injury, injury severity, and lesion location. Brain Cogn. 60, 208-2009.

Levin, H. S., Culhane, K. A., Fletcher, J. M., Mendelsohn, D. B., Lilly, M. A., Harward, H., Chapman, S. B., Bruce, D. A., Bertolino-Kusnerik, L., and Eisenberg, H. M. (1994). Dissociation between delayed alternation and memory after pediatric head injury: relationship to MRI findings. J. Child Neurol. 9, 81-89.

Levin, H. S., Culhane, K. A., Mendelsohn, D., Lilly, M. A., Bruce, D., Fletcher, J. M., Chapman, S. B., Harward, H., and Eisenberg, H. M. (1993). Cognition in relation to magnetic resonance imaging in head-injured children and adolescents. Arch. Neurol. 50, 897-905.

Levin, H. S., and Hanten, G. (2005). Executive functions after traumatic brain injury in children. Pediatr. Neurol. 33, 79-93.

Levin, H. S., Hanten, G., Chang, C. C., Zhang, L., Schachar, R., Ewing-Cobbs, L., and Max, J. E. (2002). Working memory after traumatic brain injury in children. Ann. Neurol. 52, 82-88.

Levin, H. S., Hanten, G., and Li, X. (2009). The relation of cognitive control to social outcome after paediatric TBI: Implications for intervention. Dev. Neurorehabil. 12, 320-329.

Levin, H. S., Hanten, G., Roberson, G., Li, X., Ewing-Cobbs, L., Dennis, M., Chapman, S., Max, J. E., Hunter, J., Schachar, R., Luerssen, T. G., and Swank, P. (2008). Prediction of cognitive sequelae based on abnormal computed tomography findings in children following mild traumatic brain injury. J. Neurosurg. Pediatr. 1, 461-470.

Levin, H. S., Hanten, G., Zhang, L., Swank, P. R., Ewing-Cobbs, L., Dennis, M., Barnes, M. A., Max, J., Schachar, R., Chapman, S. B., and Hunter, J. V. (2004). Changes in working memory after traumatic brain injury in children. Neuropsychology 18, 240-247.

Levin, H. S., Mendelsohn, D., Lilly, M., Yeakley, J., Song, J. X., Scheibel, R. S. Harward, H., Fletcher, J. M., Kufera, J. A., Davidson, K. C., and Bruce, D. (1997). Magnetic resonance imaging in relation to functional outcome of pediatric closed head injury: a test of the Ommaya-Gennarelli model. Neurosurgery 40, 432-440.

Luo, Q., Perry, C., Peng, D., Jin, Z., Xu, D., Ding, G., Xu, S. (2003). The neural substrate of analogical reasoning: an fMRI study. Brain Res. Cogn. Brain Res. 17, 527-534. 
Markman, A. B., and Gentner, D. (1993). Structural alignment during similarity comparisons. Cogn. Psychol. 25, 431-467.

McCauley, S.R., Wilde, E.A., Merkley, T.L., Schnelle, K.P., Bigler, E.D., Hunter, J.V., Chu, Z., Vásquez, A.C., and Levin, H.S. (2010). Patterns of cortical thinning in relation to event-based prospective memory performance three months after moderate to severe traumatic brain injury in children. Dev. Neuropsychol. 35, 318-332.

Merkley, T. L., Bigler, E. D., Wilde, E. A., McCauley, S. R., Hunter, J. V., and Levin, H. S. (2008). Diffuse changes in cortical thickness in pediatric moderate-to-severe traumatic brain injury. J. Neurotrauma 25, 1343-1345.

Morrison, R. G., Krawczyk, D. C., Holyoak, K. J., Hummel, J. E., Chow, T. W., Miller, B. L., and Knowlton, B. J. (2004). A neurocomputational model of analogical reasoning and its breakdown in frontotemporal lobar degeneration. J. Cogn. Neurosci. 16, 260-271.

Morrison, R. G., and Soohyun, C. (2008). Neurocognitive process constraints on analogy: What changes to allow children to reason like adults? Behav. Brain Sci. 31, 391-392.

Morsanyi, K. and Holyoak, K. J. (2010). Analogical reasoning ability in autistic and typically-developing children. Developmental Science 13, 578-587.

Muscara, F., Catroppa, C., and Anderson, V. (2008). The impact of injury severity on executive function 7-10 years following pediatric traumatic brain injury. Dev. Neuropsychol. 33, 623-636.

Newsome, M. R., Steinberg, J. L., Scheibel, R.S., Troyanskaya, M., Chu, Z., Hanten, G., Lu, H., Lane, S., Lin, X., Hunter, J. V., Vasquez, C., Zientz, J., Li, X., Wilde, E. A., and Levin, H. S. (2008). Effects of traumatic brain injury on working memory-related brain activation in adolescents. Neuropsychology 22, 419-425.

O’Hare, E., Whitaker, K., Op De Macks, Z., Johnson, B., Ferrer, E., and Bunge, S. (2009). Mapping relationships between cortical thickness and fluid reasoning in typically developing children and adolescents. J. Cogn. Neurosci. (supplemental) 36, 46-51.

O’Keeffe, F. M., Dockree, P. M., Moloney, P., Carton, S., and Robertson, I. H. (2007). Characterising error-awareness of attentional lapses and inhibitory control failures in patients with traumatic brain injury. Exp. Brain Res. 180, 59-67.
Oldfield, R.C. (1971). The assessment and analysis of handedness: the Edinburgh inventory. Neuropsychologia 9, 97-113.

Penn, D. C., Holyoak, K. J., and Povinelli, D. J. (2008). Darwin's mistake: explaining the discontinuity between human and nonhuman minds. Behav. Brain Sci. 31, 109-130; discussion 130-178.

Rattermann, M. J., and Gentner, D. (1998). More evidence for a relational shift in the development of analogy: children's performance on a causal-mapping task. Cogn. Dev. 13, 453-478.

Raven, J.C. (1938). Progressive Matrices: A Perceptual Test of Intelligence. London: H.K. Lewis.

Richland, L. E., Morrison, R. G., and Holyoak, K. J. (2006). Children's development of analogical reasoning: insights from scene analogy problems. J. Exp. Child Psychol. 94, 249-273.

Rosas, H. D., Liu, A. K., Hersch, S., Glessner, M., Ferrante, R. J., Salat, D. H., van der Kouwe, A., Jenkins, B. G., Dale, A. M., and Fischl, B. (2002) Regional and progressive thinning of the cortical ribbon in Huntington's disease. Neurology 58, 695-701.

Saatman, K. E., Duhaime, A. C., Bullock, R., Maas, A. I., Valadka,A., and Manley, G. T. (2008). Classification of traumatic brain injury for targeted therapies. J. Neurotrauma 25, 719-738.

Salat, D. H., Buckner, R. L., Snyder, A. Z., Greve, D. N., Desikan, R. S., Busa, E., Morris, J.C., Dale, A. M., and Fischl, B. (2004). Thinning of the cerebral cortex in aging. Cereb. Cortex 14, 721-730.

Salvucci, D. D., and Anderson, J. R. (2001). Integrating analogical mapping and general problem solving: the path-mapping theory. Cogn. Sci. 25, 67-110.

Schachar, R., Levin, H.S., Max, J.E., Purvis, K., and Chen, S. (2004). Attention deficit hyperactivity disorder symptoms and response inhibition after closed head injury in children: do preinjury behavior and injury severity predict outcome? Dev. Neuropsychol. 25, 179-198.

Segonne, F., Dale, A. M., Busa, E., Glessner, M., Salat, D. H., Hahn, H. K., and Fischl, B. (2004). A hybrid approach to the skull stripping problem in MRI. Neuroimage 22, 1060-1075.

Segonne, F., Pacheco, J., and Fischl, B. (2007). Geometrically accurate topology-correction of cortical surfaces using nonseparating loops. IEEE Trans. Med. Imaging 26, 518-529.

Shaw, P., Kabani, N. J., Lerch, J. P., Eckstrand, K., Lenroot, R., Gogtay, N.,
Greenstein, D., Clasen, L., Evans, A. Rapoport, J. L., Giedd, J. N., and Wise, S. P. (2008). Neurodevelopmental trajectories of the human cerebral cortex. J. Neurosci. 28: 3586-3594.

Smith, R., Keramatian, K., and Christoff, K. (2007). Functionally localizing the rostrolateral prefrontal cortex. Neuroimage 36, 1387-1396.

Sowell, E. R., Thompson, P. M., Holmes, C. J., Jernigan, T. L., and Toga, A. W. (1999). In vivo evidence for post-adolescent brain maturation in frontal and striatal regions. Nat. Neurosci. 2 , 859-861.

Sternberg, R. J., and Rifkin, B. (1979). The development of analogical reasoning processes. J. Exp. Child Psychol. 27, 195-232.

Taylor, H. G., Yeates, K. O., Wade, S. L., Drotar, D., Stancin, T., and Minich, N. (2002). A prospective study of short- and long-term outcomes after traumatic brain injury in children: behavior and achievement. Neuropsychology 16, 15-27.

Teasdale, G., and Jennett, B. (1974). Assessment of coma and impaired consciousness. A practical scale. Lancet 2, 81-84.

Tohill, J. M., and Holyoak, K. J. (2000). The impact of anxiety on analogical reasoning. Think. Reason. 6, 27-40.

Viskontas, I. V., Morrison, R. G., Holyoak, K. J., Hummel, J.E., and Knowlton, B. J. (2004). Relational integration, inhibition, and analogical reasoning in older adults. Psychol. Aging 19, 581-591.

Waltz, J. A., Knowlton, B. J., Holyoak, K. J., Boone, K. B., Back-Madruga, C., McPherson, S., Masterman, D. Chow, T., Cummings, J. L., Miller, B. L. (2004). Relational integration and executive function in Alzheimer's disease. Neuropsychology 18, 296-305.

Waltz, J.A., Knowlton, B. J., Holyoak, K. J., Boone, K. B., Mishkin, F. S., de Menezes Santos, M., Thomas, C. R., and Miller, B. L. (1999). A system for relational reasoning in human prefrontal cortex. Psychol. Sci. 10, 119-125.

Waltz, J. A., Lau, A., Grewal, S. K., and Holyoak, K. J. (2000). The role of working memory in analogical mapping. Mem. Cogn. 28, 1205-1212.

Wendelken, C., Nakhabenko, D. Donohue, S. E., Carter, C. S., Bunge, S. A. (2008). "Brain is to thought as stomach is to ??": investigating the role of rostrolateral prefrontal cortex in relational reasoning. J. Cogn. Neurosci. 20, 682-693.

Wharton, C. M., Grafman, J., Flitman, S S., Hansen, E. K., Brauner, J., Marks, A.,
Honda, M. (2000). Toward neuroanatomical models of analogy: A positron emission tomography study of analogical mapping. Cognitive Psychology. 40, 173-197.

Wilde, E. A., Hunter, J. V., Newsome, M. R., Scheibel, R. S., Bigler, E. D., Johnson, J. L., Fearing, M. A., Cleavinger, H. B., Li, X., Swank, P. R., Pedroza, C., Roberson, G. S., Bachevalier, J., and Levin, H. S. (2005). Frontal and temporal morphometric findings on MRI in children after moderate to severe traumatic brain injury. J. Neurotrauma 22, 333-344.

Wright, S. B., Matlen, B. J., Baym, C. L., Ferrer, E., Bunge, S. A. (2007). Neural correlates of fluid reasoning in children and adults. Front. Hum. Neurosci. 1, 8

Yeates, K. O., Bigler, E. D., Dennis, M. Gerhardt, C.A., Rubin, K. H., Stancin, T., Taylor, H. G., and Vannatta K. (2007). Social outcomes in childhood brain disorder: a heuristic integration of social neuroscience and developmental psychology. Psychol. Bull. 133 535-556.

Yeates, K. O., Taylor, H. G., Wade, S. L., Drotar, D., Stancin, T., and Minich N. (2002). A prospective study of short- and long-term neuropsychological outcomes after traumatic brain injury in children. Neuropsychology 16 , 514-523.

Yntema, D. B., and Schulman, G. M. (1969). Response selection in keeping track of several things at once. Acta Psychol. 27, 325-32.

Conflict of Interest Statement: The authors declare that the research was conducted in the absence of any commercial or financial relationships that could be construed as a potential conflict of interest.

Received: 19 April 2010; paper pending published: 08 May 2010; accepted: 15 July 2010; published online: 19 August 2010. Citation: Krawczyk DC, Hanten G, Wilde EA, LiX, Schnelle KP, Merkley TL, Vasquez AC, Cook LG, McClelland M, Chapman SB and Levin HS (2010) Deficits in analogical reasoning in adolescents with traumatic brain injury. Front. Hum. Neurosci. 4:62. doi: 10.3389/fnhum.2010.00062 Copyright (c) 2010 Krawczyk, Hanten, Wilde, Li, Schnelle, Merkley, Vasquez, Cook McClelland, Chapman and Levin. This is an open-access article subject to an exclusive license agreement between the authors and the Frontiers Research Foundation, which permits unrestricted use, distribution, and reproduction in any medium, provided the original authors and source are credited. 